\title{
Social cognition and social functioning in people with borderline personality disorder and their first-degree relatives
}

\author{
Esther Ortega-Díaz ${ }^{1}$, Jonatan García-Campos ${ }^{2}$, José M Rico-Gomis ${ }^{1,3}{ }^{\text {, }}$, Carlos Cuesta-Moreno ${ }^{1}$, Antonio Palazón- \\ Bru $^{\text {Corresp., } 3}{ }^{\text {, Gabriel Estany-Cerezo }}{ }^{4}$, José A Piqueras-Rodríguez ${ }^{5}$, Jesús Rodríguez-Marín ${ }^{5}$ \\ 1 Department of Psychiatry, General University Hospital of Elche, Elche, Alicante, Spain \\ 2 Department of Behavioral and Health Sciences, Miguel Hernández University, San Juan de Alicante, Alicante, Spain \\ 3 Department of Clinical Medicine, Miguel Hernández University, San Juan de Alicante, Alicante, Spain \\ 4 Department of Investigation, General University Hospital of Elche, Elche, Alicante, Spain \\ 5 Department of Health Psychology, Miguel Hernández University, San Juan de Alicante, Alicante, Spain \\ Corresponding Author: Antonio Palazón-Bru \\ Email address: antonio.pb23@gmail.com
}

Background. A few papers studying healthy, first-degree relatives of people with borderline personality disorder (BPD) have found that this group presents attention and memory problems. However, current research has not analyzed their social cognition. Materials \& Methods. We designed an age-, gender-, and education-level matched casecontrol study involving 57 people with BPD, 32 of their first-degree relatives, and 57 healthy controls in Spain in 2018-2019. All were assessed for social cognition and functioning using the Movie for Assessment of Social Cognition and the Social Functioning Scale; other potential confounders were also collected (marital status, occupation, and household variables). Results. There were differences in the social cognition domain of overmentalizing errors, with the BPD group scoring significantly higher than controls; however, there was no significant difference with relatives; in the social functioning domain of family relationships, with the controls showing the highest scores. Social engagement/withdrawal, interpersonal behavior, independence-competence, prosocial activities, full scale and categorization domains showed the same pattern: the BPD group had lower scores than their relatives and the controls. Relatives were significantly different from BPD patients in family relationships, social engagement/withdrawal and interpersonal behavior, as well as on the full Social Functioning Scale (both as a linear and categorical variable). However, only controls showed differences with relatives in family relationships. Conclusions. All in all, relatives show similar levels of social cognition and functioning compared with controls, and people with BPD show some alterations in different domains of both social cognition and functioning. 


\section{AUTHOR COVER PAGE:}

2 Title: Social cognition and social functioning in people with borderline personality disorder and 3 their first-degree relatives.

4 Authors: Esther Ortega-Díaz ${ }^{\mathrm{a}}$, Jonatan García-Campos ${ }^{\mathrm{b}}$, José M Rico-Gomis ${ }^{\mathrm{a}, \mathrm{c}}$, Carlos Cuesta-

5 Moreno $^{\mathrm{a}}$, Antonio Palazón-Bru ${ }^{\mathrm{c}^{*}}$, Gabriel Estañ-Cerezo ${ }^{\mathrm{d}}$, José A Piqueras-Rodríguez ${ }^{\mathrm{e}}$, Jesús

6 Rodríguez-Marín ${ }^{\mathrm{e}}$.

7 a Department of Psychiatry, General University Hospital of Elche, Elche, Alicante, Spain.

$8{ }^{\mathrm{b}}$ Department of Behavioral and Health Sciences, Miguel Hernández University, San Juan de

9 Alicante, Alicante, Spain.

10 c Department of Clinical Medicine, Miguel Hernández University, San Juan de Alicante,

11 Alicante, Spain.

$12{ }^{\mathrm{d}}$ Department of Investigation, General University Hospital of Elche, Elche, Alicante, Spain.

13 e Department of Health Psychology, Miguel Hernández University, San Juan de Alicante, 14 Alicante, Spain.

15 *Corresponding author: Prof. Dr. Antonio Palazón-Bru, Department of Clinical Medicine,

16 Miguel Hernández University, Ctra Valencia-Alicante S/N, 03550 San Juan de Alicante,

17 Alicante, Spain. Phone: +34965919449. Fax: +34965919450. E-mail: antonio.pb23@gmail.com. 
18 ABSTRACT

19 Background. A few papers studying healthy, first-degree relatives of people with borderline

20 personality disorder (BPD) have found that this group presents attention and memory problems.

21 However, current research has not analyzed their social cognition.

22 Materials \& Methods. We designed an age-, gender-, and education-level matched case-control 23 study involving 57 people with BPD, 32 of their first-degree relatives, and 57 healthy controls in

24 Spain in 2018-2019. All were assessed for social cognition and functioning using the Movie for 25 Assessment of Social Cognition and the Social Functioning Scale; other potential confounders

26 were also collected (marital status, occupation, and household variables).

27 Results. There were differences in the social cognition domain of overmentalizing errors, with

28 the BPD group scoring significantly higher than controls; however, there was no significant

29 difference with relatives; in the social functioning domain of family relationships, with the

30 controls showing the highest scores. Social engagement/withdrawal, interpersonal behavior,

31 independence-competence, prosocial activities, full scale and categorization domains showed the

32 same pattern: the BPD group had lower scores than their relatives and the controls. Relatives

33 were significantly different from BPD patients in family relationships, social

34 engagement/withdrawal and interpersonal behavior, as well as on the full Social Functioning

35 Scale (both as a linear and categorical variable). However, only controls showed differences with 36 relatives in family relationships. 
37 Conclusions. All in all, relatives show similar levels of social cognition and functioning

38 compared with controls, and people with BPD show some alterations in different domains of

39 both social cognition and functioning.

40 KEYWORDS: Borderline Personality Disorder; Cognition; Social Adjustment; Social Behavior;

41 Family. 


\section{INTRODUCTION}

43 Borderline personality disorder (BPD) is a severe psychiatric disease that predominantly

44 manifests in young adults through a pattern of instability in interpersonal relationships, self-

45 image, and affect, along with intense impulsivity (American Psychiatric Association, 2014).

46 Epidemiological studies in the United States estimate its prevalence at $0.5 \%$ to $5.9 \%$ of the

47 population (Lenzenweger et al., 2007; Grant et al., 2008; Leichsenring et al., 2011), generating a

48 high burden for public health systems (Soeteman et al., 2008).

Social cognition refers to the abilities to perceive, interpret, and process social stimuli

that guide social interactions (Green et al., 2008). Alterations in these processes could cause

51 difficulties in identifying others' emotions, thoughts, and intentions; these problems could cause

52 different symptoms, such as intense fear of abandonment or dichotomous thinking and

53 idealization (Preißler et al., 2010). Some studies relate the diagnosis of BPD with a disturbance

54 in social cognition (Minzenberg et al., 2006; Preißler et al., 2010), although there is controversy

55 on this point, probably because of the sensitivity of the instruments used (Dziobek et al., 2006;

56 Arntz et al., 2009; Preißler et al., 2010). On the other hand, when more naturalistic methods are

57 used, like the Movie for Assessment of Social Cognition (MASC) (Dziobek et al., 2006), the

58 results more precisely support alterations in the social cognition of people with BPD (Preißler et

59 al., 2010).

Social functioning is a complex and multidimensional construct, encompassing a person's

61 ability to achieve goals and play defined social roles, as well as to take care of oneself and enjoy

62 leisure time (Mueser \& Tarrier, 1998). Some authors note that social functioning covers different

63 areas, including an individual's social cognition, skills, interactions, and behaviors (Beauchamp 
$64 \&$ Anderson, 2010). In terms of the relationship between these aspects and BDP, people with

65 BPD display lower social functioning compared to the general population (Hill et al., 2008;

66 Gunderson et al., 2011; Liebke et al., 2017), and this difference is even more pronounced in the

67 presence of other psychiatric comorbidities (Mosiołek et al., 2018).

Although few studies have investigated healthy first-degree relatives of people with BPD,

69 these family members present more attention and memory problems than the general population

70 (Ruocco, Lam \& McMain, 2014). We have not found studies that analyze social cognition in

71 first-degree relatives of people with BPD, though these studies do exist in other mental

72 pathologies like schizophrenia or bipolar disorder (Lavoie et al., 2013; Reynolds, Van Rheenen

$73 \&$ Rossell, 2014). The constant deficit in social cognition has been shown to be a characteristic

74 feature of both of these conditions, extending beyond the period of crisis and constituting an

75 endophenotypic marker in populations with a heightened genetic loading for the disorder,

76 including first-degree relatives (Santos et al., 2017). Moreover, self-perceived function

77 incapacity is increased in both people with BPD and in their first-degree relatives, although in

78 the latter group to a lesser extent and in fewer functional areas (Ruocco, Lam \& McMain, 2014).

79 We are not aware of any research studying social cognition in healthy, first-degree

80 relatives of people with BPD. Thus, our objective was to determine whether diminished social

81 cognition is a characteristic feature in the first-degree relatives of people with BPD.

83 MATERIAL \& METHODS

84 Study population 
85 The study included people with BPD, their first-degree relatives (parents or children), and

86 members of the general population without any mental illness, from health department 20

87 (Valencian Region, in the southeast of Spain). The catchment area of this health department is

88 the entire population of Elche and Santa Pola, which had a registered population of 465,119

89 inhabitants in 2018.

90 Study design and participants

91 This was a case-control study involving 146 participants: 57 with BPD, according to criteria in

92 the fifth version of the Diagnostic and Statistical Manual of Mental Disorders (DSM-5)

93 (American Psychiatric Association, 2014); 32 healthy, first-degree relatives; and 57 controls

94 matched with the BPD group for age, gender, and educational level. All participants were

95 recruited between July 2018 and March 2019.

96 People with BPD were chosen from the hospital coding database at the General

97 University Hospital of Elche (the only hospital in the health department); selected patients were

98 those registered with ICD-9-CM code 301.83 and ICD-10-CM code F60.3. Afterwards, a

99 psychiatrist determined whether the patients met the DSM-5 diagnostic criteria (American

100 Psychiatric Association, 2014), and they were contacted by phone to invite them to participate in

101 the study. If the answer was affirmative, they made an appointment at the hospital. Exclusion

102 criteria were: aged under 18 years, another diagnosis of a severe mental disorder, intellectual

103 disability, residence in a different region of Spain, refusal to participate, internment in prison, or

104 inability to contact. First-degree relatives were the BPD participants' parents or children, who

105 voluntarily agreed to take part in the study; they were excluded if they were younger than 18

106 years or had a psychiatric disorder (assessed by means of the International Neuropsychiatric 
107 Interview; Sheehan et al., 1998). If more than one relative was willing to participate, they were

108 both allowed. On the other hand, if the person with BPD did not have close relatives, if the

109 relative(s) did not want to or could not participate, or if they presented an exclusion criterion,

110 then we collected data only for the person with BPD. The controls were recruited from the

111 companions of patients in the services of surgery, internal medicine, traumatology, neurology,

112 and obstetrics. Selected controls were matched with the BPD study population for age, gender,

113 and educational level. With regard to age, three controls had an age difference of one year with

114 respect to the matched patient. They were assessed using the International Neuropsychiatric

115 Interview to rule out any psychiatric disorders (Sheehan et al., 1998).

\section{Variables and measurement}

117 Social cognition was measured by means of the Spanish version of MASC (Lahera et al., 2014).

118 This is a naturalistic measure combining auditory, verbal, and emotional channels. For its

119 administration, participants were asked to watch a short film in which four people appeared in

120 different daily situations. During the movie, they had to answer 45 multiple-choice questions

121 about the characters' feelings, thoughts, and intentions. In addition to the correct answer, there

122 were three error categories: undermentalizing errors, wherein the person has a general—but

123 underdeveloped - idea of what the other could be feeling or thinking; theory of mind absence

124 errors, which occur when there is no connection between one's observation and interpretation;

125 and overmentalizing errors; which stem from an excessive interpretation of another's state of

126 mind (Dziobek et al., 2006; Sharp et al., 2011).

127 The scores took into account both the total number of correct answers and the total errors, and

128 the latter were analyzed by subtype (Lahera et al., 2014). A lower number of correct answers 
129 indicates a worse condition. This measure presents high test-retest reliability and high internal

130 consistency in both its original English version (Dziobek et al., 2006) and its translation into

131 Spanish (Lahera et al., 2014)

132 Social functioning was measured using the Spanish version of the self-administered

133 Social Functioning Scale (SFS, Vázquez \& Jiménez, 2000). The SFS assesses seven areas of

134 social functioning in the previous three months on a scale of 70 to 129: social

135 isolation/integration ( 0 to 15$)$, interpersonal communication ( 0 to 9$)$, pro-social activities ( 0 to

136 48), recreation (0 to 32), independence-competence (13 to 39), independence-performance (0 to

137 39), and employment/occupation (0 to 129). The score cutoffs group respondents in three

138 categories: low-functioning $(<95)$, medium-functioning (95-106), and high-functioning $(>106)$.

139 Two versions of the scale exist, depending on the information source used to understand the

140 patient's social functioning: in the self-report version, patients themselves complete the

141 questionnaire and provide information on their behavior; and in the informant report, relatives

142 take on this role. Because we aimed to analyze all of the participants' behavior (people with

143 BPD, relatives, and controls), we opted to use the self-report version. The English version shows

144 adequate reliability, validity, and sensitivity (Birchwood et al., 1990). In the Spanish version,

145 internal consistency and test-retest reliability demonstrate some variability, but the results are

146 still satisfactory (Vázquez \& Jiménez, 2000).

147 In addition, the following variables were collected: gender, age (in years), highest

148 educational level attained (primary school, secondary school, and university), marital status

149 (single, married/with stable partner, and separated/widower), occupation (active, unemployed, 
150 sick leave/pensioner, and student) and household composition (own family, family of origin, and 151 single).

152 Participants were convened in groups of four or fewer in the classroom space at the

153 General University Hospital of Elche. The facilities had a projector, screen, tables, and chairs.

154 Participants received an information sheet on the study and signed informed consent. They then

155 underwent assessments with the MASC and SFS - always in that order and administered by the

156 same professional, a psychiatric occupational therapist with more than 10 years of experience in

157 the service.

158 Sample size

159 The sample size was calculated to compare mean scores on the SFS scale among the three groups

160 (ANOVA). To estimate the means in each group, we randomly selected $15 \%$ of the total sample,

161 obtaining the following values: 114.4 for the control group; 115.9 for the relatives; and 101.3 for

162 the BPD group. The estimated standard deviation (SD) was 10.9. According to these parameters

163 and using a type I and type II error of 5\%, we calculated a minimum number of 15 participants

164 per group (Chow, Wang \& Shao, 2008).

165 Statistical methods

166 Qualitative variables were described as absolute and relative frequencies, while quantitative

167 variables were expressed as means (SD) or medians (interquartile range). To compare group

168 characteristics, Pearson's chi-squared and ANOVA tests were applied. To assess differences in

169 the scales administered to the three groups, median or ANOVA tests were used, depending on

170 whether the variable of interest was continuous or discrete. Post-hoc analysis was carried out 
171 using the Bonferroni correction. For the multivariable analysis, linear or ordinal quantile

172 (median) regression models were fitted to adjust the results for marital status, occupation, and

173 household composition. All analyses were performed at a significance level of 5\%, and

174 confidence intervals (CIs) were calculated for each relevant parameter. The statistical software

175 used was IBM SPSS Statistics 25 and R 3.5.1.

\section{Ethical considerations}

177 Both the Research Commission and the Research Ethics Committee at the General University

178 Hospital of Elche approved the study (25 June 2018 and 26 June 2018, respectively). All

179 participants were adequately informed of the study aims and methods, and if they agreed to take 180 part, they signed informed consent before their inclusion.

\section{RESULTS}

183 A total of 146 participants were included: 57 had a diagnosis of BPD, 32 were first-degree

184 relatives of these people, and 57 were healthy controls. Tables 1-2 show the demographic

185 characteristics of the three groups. Most of the participants in the BPD and control groups were

186 women $(91.2 \%)$, while a smaller majority were women in the relatives group $(62.5 \% ; \mathrm{p}<0.001$

187 in the total comparison and $\mathrm{p}=0.002$ when we compared relatives versus controls or patients).

188 Mean age was 33.4 years in the BPD and control groups, and it was 52.9 in the group of first-

189 degree relatives $(\mathrm{p}<0.001$ in both the global analysis and the comparison of relatives with BPD

190 versus controls). Cases and controls showed a similar educational level ( $\mathrm{p}>0.0056)$, with most

191 having completed secondary school, while the relatives showed a lower level $(\mathrm{p}=0.003$ versus 
192 controls and $\mathrm{p}=0.005$ versus patients in secondary school). There were also differences in

193 marital status $(\mathrm{p}=0.006)$, occupation $(\mathrm{p}<0.001)$, and household composition $(\mathrm{p}=0.004)$.

194 Specifically, the relatives were less likely to be single than patients $(p=0.002)$, more likely to be

195 on disability or pension rolls than controls $(\mathrm{p}<0.001)$, and less likely to be living with their

196 family of origin compared with patients $(p=0.001)$.

197 Bivariable analysis of the questionnaires among the three groups (Tables 3-4) showed

198 statistically significant differences $(\mathrm{p}<0.05)$ in the number of correct MASC items $(\mathrm{p}=0.012)$

199 and the MASC Overmentalizing errors $(\mathrm{p}=0.006)$. Controls scored higher on the first and lower

200 on the second; relatives did not present statistical differences with the other groups $(\mathrm{p}>0.017)$.

201 All SFS dimensions showed differences as well $(\mathrm{p}<0.05)$, with the relatives group presenting

202 higher scores than the BPD group. However, the only dimensions reaching statistical differences

203 between relatives and BPD patients $(p<0.017)$ were social engagement/withdrawal $(p<0.001)$,

204 independence-performance $(\mathrm{p}=0.008)$ and recreation $(\mathrm{p}=0.008)$. In contrast, relatives showed

205 significantly higher overall scores on the SFS than BPD patients $(p<0.001)$ and similar scores to

206 the control group $(\mathrm{p}=0.198)$. When the variable was categorized, likewise there was a

207 significantly larger proportion of relatives compared to BPD patients with higher scores $(\mathrm{p}=$ 208 0.001).

Table 5 shows the results of the multivariable analysis, assessing differences between

210 groups in the questionnaires used, but adjusting for marital status, occupation, and household

211 composition. After controlling for these confounders, using the relatives group as a reference,

212 there were no significant differences in the MASC subscales. On the family relationships

213 subscale of the SFS, the controls scored the highest $(2, \mathrm{p}<0.001)$, followed by the relatives $(0)$ 
214 and finally the cases $(-2, p=0.014)$. The SFS subscales of social engagement/withdrawal,

215 interpersonal behavior, independence-competence, and prosocial activities, plus the full scale

216 and the categorization, showed the same pattern, with the BPD group achieving lower scores

217 than the relatives and the controls. However, the statistical differences $(p<0.05)$ were in: social

218 engagement/withdrawal (relatives vs BPD, $p=0.002$ ), interpersonal behavior (relatives vs BPD,

$219 \mathrm{p}=0.019)$ ), SFS full scale (relatives vs BPD, $\mathrm{p}<0.001$ ) and categorized SFS scores (relatives vs

220 BPD, $\mathrm{p}=0.003)$.

\section{DISCUSSION}

\section{Summary}

224 The present study investigated social cognition and functioning in people with BPD, their

225 healthy, first-degree relatives, and a group of healthy controls. Our results show that relatives of

226 people with BPD show some alterations in social cognition; however, results were not

227 statistically significant, so there is insufficient evidence to support that this is a characteristic

228 feature of BPD. With regard to social functioning, first-degree relatives showed a significant

229 deficit on the family relationships subscale compared to controls and BPD patients, and in social

230 engagement/withdrawal and interpersonal behavior compared with patients. We observed similar

231 results when assessing the SFS full scale, as both a linear and categorical variable.

\section{Strengths and limitations}


233 The main strength of our study is its novel nature; we did not find any other paper in the

234 literature examining social cognition in first-degree relatives of people with BPD. In addition,

235 the statistical power was over $95 \%$ for testing differences in means in the calculation of the

236 sample size, which increases the precision of our results.

To minimize selection bias, the sampling frame was the list of patients in the hospital

238 coding database, not those attending the consult, as in previous studies (Minzenberg, Poole \&

239 Vinogradov, 2006). This is an important issue, as it increases the representativeness of the

240 sample of people with BPD. Furthermore, the controls were selected using a population-based

241 approach from the same geographical area, and they did not have any other pathology, especially

242 mental disorders. With regard to information bias, data collection was undertaken by a single

243 professional with experience administering the questionnaires used in this study, which enhances

244 the reliability of the results obtained. Other studies have not taken the same precautions to limit

245 this form of bias (Lahera et al., 2014). In addition, we used internationally validated scales;

246 MASC (Dziobek et al., 2006) is much more naturalistic and precise than other measurement

247 instruments. Moreover, by estimating the magnitude of effects through multivariable models, we

248 could minimize the risk of confounding bias, as evidenced by the loss of statistical significance

249 between the bivariable and the multivariable analysis after adjusting for other factors. On the

250 other hand, it was not feasible to match the relatives for gender, age, or educational level,

251 therefore we cannot rule out the influence of confounding in this group. Furthermore, we could

252 not guarantee that the patients with BPD were in the same disease stage during assessment,

253 which could alter the results and should be taken into account in future studies. Finally, we were

254 unable to determine whether the origin of the alterations of function and the perception of 
255 emotions were due to genetic or environmental factors, for example living with a person that had 256 BPD.

257 Comparison with existing literature

258 We did not find any paper that assessed social cognition in first-degree family members of 259 people with BPD, although there are similar studies in other mental disorders, like bipolar 260 disorder and schizophrenia, that have reported alterations. In the case of schizophrenics, their 261 relatives did not show important deficits in social cognition, but they did show lower

262 performance than the general population (Lavoie et al., 2013; Reynolds et al., 2014). These

263 results, together with the literature reporting that alterations in social cognition can be observed

264 in patients "in remission" (Bora, Yucel \& Pantelis, 2009) support the hypothesis that social

265 cognition capacities may be related to a disorder's genetic component (Gottesman \& Gould, 266 2003), and deficits in these processes could stem from genetic vulnerability in BPD (Lavoie et 267 al., 2013; Reynolds et al., 2014).

Our results differ from those of other studies that have not found any diminishment of social cognition in people with BPD, for example in Preißler et al.'s (2010) study, which used the "Reading the Mind in the Eyes" test (RME), or Arntz et al.'s (2009) paper, which used the

271 advanced test of theory of mind. On the other hand, Minzenberg et al. (2006) used the Buss-

272 Durkee hostility index, finding a normal capacity for recognizing isolated facial or prosodic

273 emotions but difficulties in recognizing integrated ones. These differences could be due to the 274 psychometric tools used, as naturalistic scales like MASC (Dziobek et al., 2006) yield more 275 precise results. Other groups that have used MASC, like Preißler et al., (2010) and Sharp et al., 276 (2011), have reported similar results to ours, with BPD patients showing alterations in social 
277 cognition. We obtained higher scores in social cognition in the form of overmentalization errors,

278 which coincides with Sharp et al.'s (2011) study in a sample of adolescents with borderline

279 features.

With regard to social functioning, our results differ from Ruocco et al.'s (2014), where

relatives reported greater functional limitations than controls in life activities and participation in society. However, in our study, despite the lower scores achieved by relatives compared to controls in some domains, significant differences were only apparent in family relationships.

284 Likewise, we found small but significant differences in our BPD group in the domains of social 285 engagement/withdrawal, interpersonal behavior, independence-competence, prosocial activities, and on the full-scale SFS, similarly to Ruocco et al., (2014), who reported that the probands showed higher levels of incapacity than their relatives and the controls in all functional domains: comprehension and communication, mobility, self-care, interpersonal relations, life activities

289 (domestic, leisure, work, and academic activities), and participation in society. Finally, Liebke et al., (2016) used the same social functioning scale as we did, although their sample did not

291 include first-degree relatives. Patients with BPD showed low social functioning, while in our 292 sample they presented medium functioning. Likewise, in their study there were significant 293 alterations in all domains in BPD participants compared to controls, while in our study the 294 differences were not significant in the domains of recreation or employment/occupation. These differences could be due to the distinct cultural characteristics, as their study took place in

296 Germany, while ours was in Spain. Finally, Skodol et al., (2005) used the Longitudinal Interval 297 Follow-up Evaluation, and they found significant deficits in the domains of interpersonal 298 behavior, prosocial activities, full scale, and occupation. However, it is difficult to draw a comparison with our results because of the different measures used. 
301 We detected diminished social cognition skills in people with BPD, along with limitations in

302 some domains of social functioning in both the people with BPD and in their first-degree

303 relatives. These results could support development of interventions to reduce the deficits

304 identified. In light of our findings, future studies are needed to determine whether a deficit in the

305 domain of family relationships in healthy relatives influences the social functioning, social

306 cognition, and/or the symptomology of people with BPD. Additional research is also needed to

307 understand the pathophysiology of BPD, including the role of genetic and socioenvironmental 308 factors.

\section{CONCLUSIONS}

311 Our results show that healthy first-degree relatives of people with BPD present similar social

312 cognition skills as healthy controls, with no genetic vulnerability related to BPD. The social

313 cognition of people with this disorder demonstrates greater deficits in the form of

314 overmentalization. Compared to patients, relatives showed significant differences in social

315 functioning with regard to family relationships, social engagement/withdrawal and interpersonal

316 behavior, and compared to controls, relatives showed differences in family relationships.

317 Otherwise, social functioning is quite similar between relatives and controls, while people with

318 BPD show lower social functioning across many domains. 


\section{ACKNOWLEDGMENTS}

321 The authors thank Meggan Harris for her help in the English version of the text. Furthermore, we

322 would thank the contribution of Guillermo La Hera Forteza for providing us with relevant

323 information about the MASC scale validation. 
324 REFERENCES

325 American Psychiatric Association. 2014. Manual Diagnóstico y Estadístico de los Trastornos

326 Mentales (DSM-5). Madrid, Spain: Panamericana.

327 Arntz A, Bernstein D, Oorschot M, Schobre, P. 2009. Theory of mind in borderline and cluster-C

328 personality disorder. The Journal of Nervous and Mental Disease 197:801-807.

329 Beauchamp MH, Anderson V. 2010. SOCIAL: An integrative framewok for the development of

330 social skills. Psychological Bulletin 136:39-64.

331 Birchwood M, Smith J, Cochrane R, Wetton S, Copestake S. 1990. The Social Functioning

332 Scale: the development and validation of a scale of social adjustment for use in family

333 intervention programmes with schizophrenic patients. The British Journal of Psychiatry

$334 \quad 157: 853-859$.

335 Bora E, Yucel M, Pantelis C. 2009. Cognitive endophenotypes of bipolar disorder: a meta-

336 analysis of neuropsychological deficits in euthymic patients and their first-degree relatives.

337 Journal of Affective Disorders 113:1-20.

338 Chow S, Wang H, Shao J. 2008. Sample size calculations in Clinical research. Second ed. New

339 York: Chapman \& Hall /CRC.

340 Conselleria de Sanidad Universal y Salud Pública. Portal del Departamento de Salud de Elche-

341 Hospital General. 2018. Departamento de Salud Elche Hospital General. Memoria de actividad

342 2018. Avaible at 
343 http://www.elche.san.gva.es/documents/3362585/3454912/MEMORIA+2018+.pdf. (accessed: 1

344 March 2019).

345 Dziobek I, Fleck S, Kalbe E, Rogers K, Hassenstab J, Brand M, Kessler J, Woike JK, Wolf OT, 346 Convit A. 2006. Introducing MASC: a movie for the assessment of social cognition. Journal of

347 Autism and Developmental Disorders 36:623-636.

348 Gottesman II, Gould TD. 2003. The endophenotype concept in psychiatry: etymology and 349 strategic intentions. American Journal of Psychiatry 160:636-45.

350 Grant BF, Chou SP, Goldstein RB, Huang B, Stinson FS, Saha TD, Smith SM, Dawson DA, 351 Pulay AJ, Pickering RP, Ruan WJ. 2008. Prevalence, correlates, disability, and comorbidity of 352 DSM-IV borderline personality disorder: results from the Wave 2 National Epidemiologic 353 Survey on Alcohol and Related Conditions. Journal of Clinical Psychiatry 69:533-545.

354 Green MF, Penn DL, Bentall R. Carpenter WT, Gaebel W, Gur RC, Kring AM, Park S, 355 Silverstein SM, Heinssen R. 2008. Social cognition in schizophrenia: an NIMH workshop on 356 definitions, assessment, and research opportunities. Schizophrenia Bulletin 34(6):1211-20.

357 Gunderson JG, Stout RL, McGlashan TH, Shea MT, Morey LC, Grilo CM, Zanarini MC, Yen S, 358 Markowitz JC, Sanislow C, Ansell E, Pinto A, Skodol AE. 2011. Ten-year course of borderline 359 personality disorder: psychopathology and function from the Collaborative Longitudinal 360 Personality Disorders study. Archives of General Psychiatry 68:827-37.

361

362 Hill J, Pilkonis P, Morse J, Feske U. 2008. Social domain dysfunction and disorganization in 363 borderline personality disorder. Psychological Medicine 38:135-146. 
364 Lahera G, Boada L, Pousa E, Mirapeix I, Morón-Nozaleda G, Marinas L, Gisbert L, Pamiàs M,

365 Parellada M. 2014. Movie for the Assessment of Social Cognition (MASC): Spanish Validation.

366 Journal of Autism and Developmental Disorders 44:1886-1896.

367 Lavoie MA, Plana I, Lacroix JB, Godmaire-Duhaime, F, Jackson PL, Achim AM. 2013. Social

368 cognition in first-degree relatives of people with schizophrenia: A meta-analysis. Psychiatry

369 Research 209:129-35.

370 Leichsenring F, Leibing E, Kruse J, New AS, Leweke F. 2011. Borderline personality disorder.

371 The Lancet 377:74-84.

372 Lenzenweger MF, Lane MC, Loranger AW, Kessler RC. 2007. DSM-IV personality disorders in 373 the National Comorbidity Survey Replication. Biological Psychiatry 62:553-564.

374 Liebke L, Bungert M, Thome, J, Hauschild S. 2017. Loneliness, social networks, and social 375 functioning in borderline personality disorder. Personality Disorders 8:349-356.

376 Minzenberg MJ, Poole JH, Vinogradov S. 2006. Social-emotion recognition in borderline 377 personality disorder. Comprehensive Psychiatry 47:468-474.

378 Mosiołek A, Gierus J, Koweszko T, Szulc, A. 2018. Evaluation of the relationship between 379 cognitive functioning in patients with borderline personality disorder and their general 380 functioning. Psychiatria Polska 28:33-44.

381 Mueser KT, Tarrier N. 1998. Handbook of social functioning in Schizophrenia. Boston: Allyn \& 382 Bacon. 
383 Preißler S, Dziobek I, Ritter K, Heekeren HR, Roepke S. 2010. Social cognition in borderline

384 personality disorder: evidence for disturbed recognition of the emotions, thoughts, and intentions

385 of other. Frontiers in Behavioral Neuroscience 2:1-12.

386 Reynolds MT, Van Rheenen TE, Rossell SL. 2014. Theory of mind in first degree relatives of

387 individuals with bipolar disorder. Psychiatry Research 219:400-402.

388 Ruocco AC, Lam J, McMain SF. 2014. Subjective cognitive complaints and functional disability

389 in patients with borderline personality disorder and their nonaffected first-degree relatives. The

390 Canadian Journal of Psychiatry 59:335-44.

391

392 Santos JM, Pousa E, Soto E, Comes A, Roura P, Arrufat FX, Obiols JE. Theory of Mind in 393 euthymic bipolar patients and first-degree relatives. The Journal of Nervous and Mental Disease 394 205:207-212.

395

396 Sharp C, Pane H, Ha C, Venta A, Patel AB, Sturek J, Fonagy P. Theory of mind and emotion 397 regulation difficulties in adolescents with borderline traits. Journal of the American Academy of 398 Child and Adolescent Psychiatry 50:563-573.

399 Sheehan D, Lecrubier Y, Sheehan KH, Amorim P, Janavs J, Weiller E, Hergueta T, Baker R, 400 Dunbar GC. 1998. The Mini International Neuropsychiatric Interview (MINI): The Development 401 and Validation of a Structured Diagnostic Psychiatric Interview for DSM-IV and ICD-10. 402 Journal of Clinical Psychiatry 59:22-33. 
403 Skodol AE, Pagano ME, Bender DS, Shea MT, Gunderson JG, Yen S, Stout RL, Morey LC,

404 Sanislow CA, Grilo CM, Zanarini MC, McGlashan TH. 2005. Stability of functional impairment 405 in patients with schizotypal, borderline, avoidant, or obsessive-compulsive personality disorder 406 over two years. Psychological Medicine 35:443-451.

407 Soeteman DI, Hakkaart-van Roijen L, Verheul R, Busschbach JJ. 2008. The economic burden of 408 personality disorders in mental health care. The Journal of Clinical Psychiatry 69:259-65.

409 Vázquez AJ, Jiménez R. 2000. Social functioning scale: new contributions concerning its

410 psychometric characteristics in a Spanish adaptation. Psychiatry Research 93:247-56. 


\section{Table $\mathbf{1}$ (on next page)}

Sociodemographic factors in the three analyzed groups: people with borderline personality disorder, first-degree relatives, and controls

${ }^{\dagger}$ Unless otherwise noted. BPD, borderline personality disorder; SD, standard deviation. 
1 Table 1: Sociodemographic factors in the three analyzed groups: people with borderline

2 personality disorder, first-degree relatives, and controls

\begin{tabular}{lcccc}
\hline Variable & Controls & Relatives & BPD & \\
& $\mathbf{N = 5 7}$ & $\mathbf{N = 3 2}$ & $\mathbf{N = 5 7}$ & p-value \\
& $\mathbf{n}(\mathbf{\%})^{\dagger}$ & $\mathbf{n}(\mathbf{\%})^{\dagger}$ & $\mathbf{n}(\mathbf{\%})^{\dagger}$ & \\
\hline Women & $52(91.2)$ & $20(62.5)$ & $52(91.2)$ & $<0.001$ \\
Educational level: & & & & \\
Primary school & $17(29.8)$ & $19(59.4)$ & $18(31.6)$ & 0.027 \\
Secondary school & $33(57.9)$ & $8(25.0)$ & $32(56.1)$ & \\
$\quad$ University & $7(12.3)$ & $5(15.6)$ & $7(12.3)$ & \\
Marital status: & & & & \\
Single & $18(31.6)$ & $4(12.5)$ & $26(45.6)$ & 0.006 \\
Married/with stable partner & $35(61.4)$ & $20(62.5)$ & $25(43.9)$ & \\
Separated/widower & $4(7.0)$ & $8(25.0)$ & $6(10.5)$ & \\
Occupation: & & & & \\
Active & $37(64.9)$ & $12(37.5)$ & $16(28.1)$ & $<0.001$ \\
Unemployed & $5(8.8)$ & $4(12.5)$ & $20(35.1)$ & \\
Sick leave/pensioner & $3(5.3)$ & $11(34.4)$ & $8(14.0)$ & \\
Student & $12(21.1)$ & $5(15.6)$ & $13(22.8)$ & \\
& & & &
\end{tabular}

Household composition:

Own family

Family of origin

Single

Age (years), mean $\pm \mathrm{SD}$

$\begin{array}{cccc}40(70.2) & 25(78.1) & 24(42.1) & 0.004 \\ 13(22.8) & 4(12.5) & 22(38.6) & \\ 4(7.0) & 3(9.4) & 11(19.3) & \\ 33.4 \pm 10.7 & 52.9 \pm 16.3 & 33.4 \pm 10.7 & <0.001\end{array}$

${ }_{\dagger}$ Unless otherwise noted.

4 BPD, borderline personality disorder; SD, standard deviation. 


\section{Table 2 (on next page)}

Post-hoc analysis with the Bonferroni correction ( $p$-values) of the sociodemographic factors in the three analyzed groups: people with borderline personality disorder, firstdegree relatives, and controls

BPD, borderline personality disorder. 
1 Table 2: Post-hoc analysis with the Bonferroni correction (p-values) of the sociodemographic factors in the three analyzed groups:

2 people with borderline personality disorder, first-degree relatives, and controls

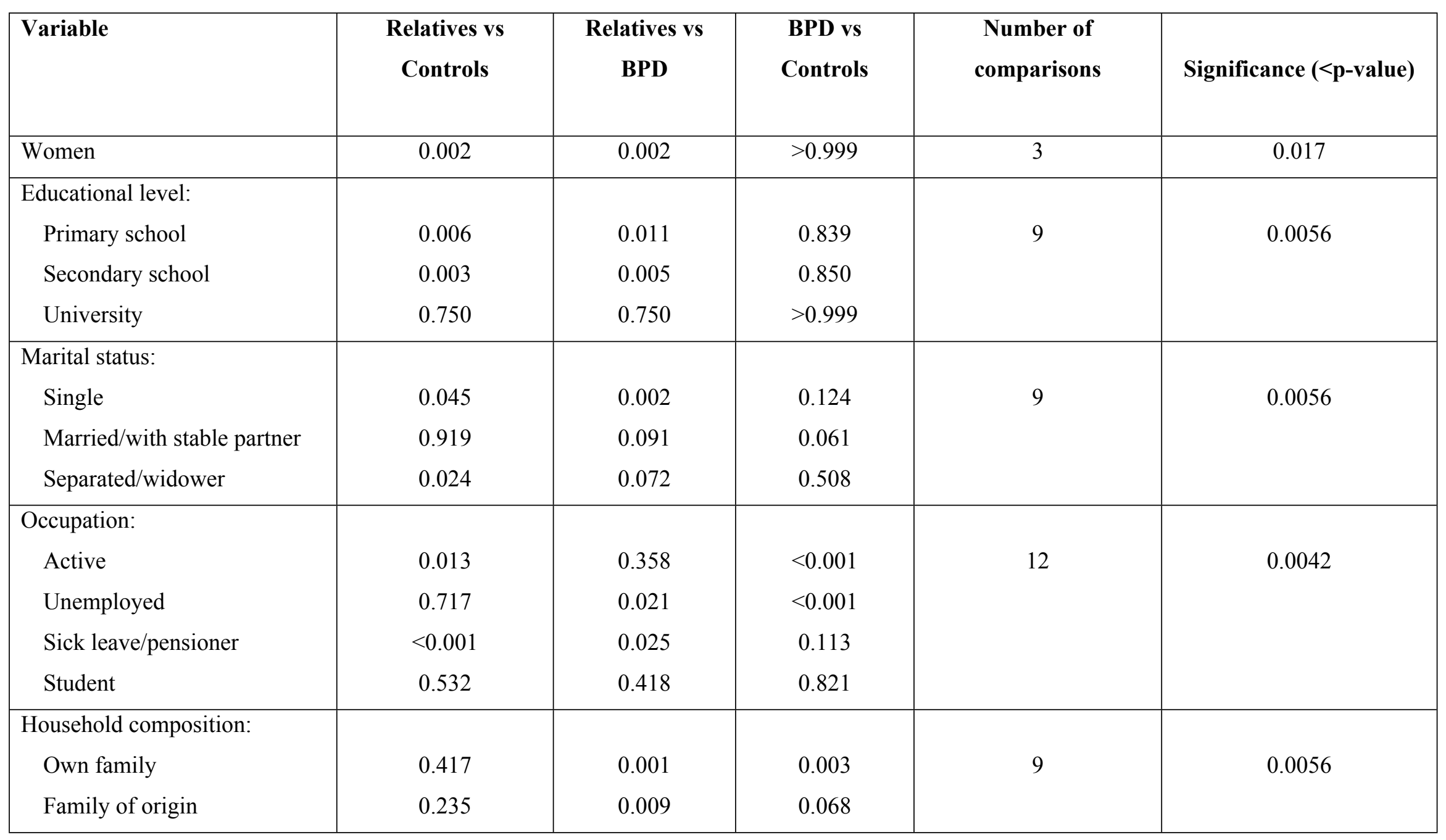




\begin{tabular}{|l|c|c|c|c|c|}
\hline \multicolumn{1}{|c|}{ Single } & 0.699 & 0.217 & 0.052 & & \\
\hline Age & $<0.001$ & $<0.001$ & $>0.999$ & 3 & 0.017 \\
\hline
\end{tabular}

3 BPD, borderline personality disorder. 


\section{Table 3(on next page)}

Scores of the scales applied in the three study groups.

${ }^{\dagger}$ It was not possible to carry out the median test due to the number of valid cases.

Abbreviations: BPD, borderline personality disorder; MASC, Movie for the Assessment of Social Cognition; IQR, interquartile range; SFS, Social Functioning Scale. 
1 Table 3: Scores of the scales applied in the three study groups.

\begin{tabular}{|c|c|c|c|c|}
\hline \multirow[t]{2}{*}{ Item } & Controls & Relatives & BPD & \multirow[t]{2}{*}{ p-value } \\
\hline & $\mathbf{N}=\mathbf{5 7}$ & $\mathrm{N}=32$ & $\mathbf{N}=\mathbf{5 7}$ & \\
\hline \multicolumn{5}{|l|}{ MASC, median (IQR) } \\
\hline Correct & $31(6)$ & $27(9)$ & $28(5)$ & 0.012 \\
\hline Overmentalizing errors & $6(4)$ & $8(6)$ & $8(4)$ & 0.006 \\
\hline Undermentalizing errors & $6(3)$ & $6(4)$ & $6(3)$ & 0.23 \\
\hline Theory of mind absence errors & 2(3) & $3(2)$ & $3(3)$ & 0.27 \\
\hline \multicolumn{5}{|l|}{ SFS domains, median (IQR) } \\
\hline Family relationships & $10(2)$ & $9(2)$ & $7(3)$ & $<0.001$ \\
\hline Social engagement/withdrawal & $13(2)$ & $12(2)$ & $9(5)$ & $<0.001$ \\
\hline Interpersonal behavior & $8(1)$ & $8(1)$ & $7(3)$ & $\dagger$ \\
\hline Independence-performance & $34(8)$ & $32(10)$ & $28(10)$ & 0.004 \\
\hline Independence-competence & $39(0)$ & $38(1)$ & $36(4)$ & $\dagger$ \\
\hline Recreation & $22(8)$ & $22(8)$ & $18(7)$ & 0.009 \\
\hline Prosocial activities & $23(11)$ & $20(12)$ & $16(16)$ & 0.011 \\
\hline Employment/occupation & $9(1)$ & $9(1)$ & $6(8)$ & $<0.001$ \\
\hline SFS full scale, mean \pm SD & $114.5 \pm 5.5$ & $112.6 \pm 8.4$ & $102.4 \pm 11.6$ & $<0.001$ \\
\hline \multicolumn{5}{|l|}{ SFS scores, $\mathrm{n}(\%)$} \\
\hline Low & $0(0)$ & $2(6.3)$ & $12(21.1)$ & $<0.001$ \\
\hline Medium & $5(8.8)$ & $4(12.5)$ & $20(35.1)$ & \\
\hline High & $52(91.2)$ & $26(81.3)$ & $25(43.9)$ & \\
\hline
\end{tabular}

$2+$ It was not possible to carry out the median test due to the number of valid cases. 
3 Abbreviations: BPD, borderline personality disorder; MASC, Movie for the Assessment of

4 Social Cognition; IQR, interquartile range; SFS, Social Functioning Scale. 


\section{Table 4 (on next page)}

Post-hoc analysis with the Bonferroni Correction ( $p$-values) for the scores of the scales applied in the three study groups.

${ }^{\dagger}$ It was not possible to carry out the median test due to the number of valid cases.

Abbreviations: BPD, borderline personality disorder; MASC, Movie for the Assessment of Social Cognition; SFS, Social Functioning Scale. 
1 Table 4: Post-hoc analysis with the Bonferroni Correction (p-values) for the scores of the scales applied in the three study groups.

\begin{tabular}{|c|c|c|c|c|c|}
\hline Item & $\begin{array}{c}\text { Relatives vs } \\
\text { Controls }\end{array}$ & $\begin{array}{l}\text { Relatives } \\
\text { vs BPD }\end{array}$ & $\begin{array}{c}\text { BPD vs } \\
\text { Controls }\end{array}$ & $\begin{array}{l}\text { Number of } \\
\text { comparisons }\end{array}$ & $\begin{array}{c}\text { Significance } \\
\text { (<p-value) }\end{array}$ \\
\hline \multicolumn{6}{|l|}{ MASC } \\
\hline Correct & 0.080 & 0.887 & 0.005 & 3 & 0.017 \\
\hline Overmentalizing errors & 0.347 & 0.842 & 0.002 & 3 & 0.017 \\
\hline \multicolumn{6}{|l|}{ SFS domains } \\
\hline Family relationships & 0.021 & 0.055 & $<0.001$ & 3 & 0.017 \\
\hline Social engagement/withdrawal & 0.709 & $<0.001$ & $<0.001$ & 3 & 0.017 \\
\hline Independence-performance & 0.832 & 0.008 & $<0.001$ & 3 & 0.017 \\
\hline Recreation & 0.104 & 0.008 & 0.005 & 3 & 0.017 \\
\hline Pro-social activities & 0.142 & 0.236 & 0.003 & 3 & 0.017 \\
\hline Employment/occupation & $\dagger$ & 0.148 & $<0.001$ & 3 & 0.017 \\
\hline SFS full scale & 0.198 & $<0.001$ & $<0.001$ & 3 & 0.017 \\
\hline SFS scores & & & & 9 & 0.0056 \\
\hline Low & 0.127 & 0.066 & $<0.001$ & & \\
\hline Medium & 0.717 & 0.021 & 0.001 & & \\
\hline
\end{tabular}



High
0.193
0.001
$<0.001$

$2 \dagger$ It was not possible to carry out the median test due to the number of valid cases.

3 Abbreviations: BPD, borderline personality disorder; MASC, Movie for the Assessment of Social Cognition; SFS, Social Functioning 4 Scale. 


\section{Table 5 (on next page)}

Multivariable analysis of the scales used in our patients, relatives and controls (coefficients with their 95\% confidence intervals).

BPD, borderline personality disorder; MASC, Movie for the Assessment of Social Cognition;

SFS, Social Functioning Scale. All the coefficients were adjusted by marital status, occupation, and household composition. 
1 Table 5: Multivariable analysis of the scales used in our patients, relatives and controls

2 (coefficients with their 95\% confidence intervals).

\begin{tabular}{|c|c|c|c|c|c|}
\hline Variable & Relatives & Controls & $\begin{array}{c}\text { p- } \\
\text { value }\end{array}$ & BPD & $\begin{array}{c}\text { p- } \\
\text { value }\end{array}$ \\
\hline \multicolumn{6}{|l|}{ Quantile regression } \\
\hline \multicolumn{6}{|l|}{ MASC: } \\
\hline Correct & 0 & $1(-3$ to 5$)$ & 0.634 & $0(-3$ to 3$)$ & $>0.999$ \\
\hline Overmentalizing errors & 0 & $-1(-4$ to 2$)$ & 0.457 & $1(-2$ to 4$)$ & 0.506 \\
\hline Undermentalizing errors & 0 & $0(-2$ to 2$)$ & $>0.999$ & $-1(-3$ to 1$)$ & 0.250 \\
\hline Theory of mind absence errors & 0 & $-0.5(-2$ to 1$)$ & 0.561 & $0(-2$ to 2$)$ & $>0.999$ \\
\hline \multicolumn{6}{|l|}{ SFS domains: } \\
\hline Family relationships & 0 & $2(1$ to 3$)$ & $<0.001$ & $-2(-4$ to 0$)$ & 0.014 \\
\hline Social engagement/withdrawal & 0 & $0(-1$ to 1$)$ & $>0.999$ & $-2.5(-4$ to -1$)$ & 0.002 \\
\hline Interpersonal behavior & 0 & $0(0$ to 0$)$ & $>0.999$ & $-1(-2$ to 0$)$ & 0.019 \\
\hline Independence-performance & 0 & $0(-3$ to 3$)$ & $>0.999$ & $-3(-7$ to 1$)$ & 0.132 \\
\hline Independence-competence & 0 & $0(0$ to 0$)$ & $>0.999$ & $-2(-4$ to 0$)$ & 0.052 \\
\hline Recreation & 0 & $-2(-6$ to 2$)$ & 0.283 & $-4(-8$ to 0$)$ & 0.083 \\
\hline Prosocial activities & 0 & $1(-6$ to 8$)$ & 0.778 & $-5(-12$ to 2$)$ & 0.189 \\
\hline Employment/occupation & 0 & $0(-1$ to 1$)$ & $>0.999$ & $-1(-3$ to 1$)$ & 0.319 \\
\hline \multicolumn{6}{|l|}{ Linear regression } \\
\hline SFS (full scale) & 0 & $-0.94(-4.7$ to 2.8$)$ & 0.627 & $-9.9(-13.7$ to -6.1$)$ & $<0.001$ \\
\hline \multicolumn{6}{|l|}{ Ordinal regression (odds ratio) } \\
\hline SFS (categorized) & 1 & $1.24(0.27$ to 5.76$)$ & 0.780 & $0.14(0.04$ to 0.52$)$ & 0.003 \\
\hline
\end{tabular}

3 BPD, borderline personality disorder; MASC, Movie for the Assessment of Social Cognition;

4 SFS, Social Functioning Scale.

5 All the coefficients were adjusted by marital status, occupation, and household composition. 